(C) EUSP, 2017 ej $\quad$ ISSN 2310-3817 Vol.5 SUCSIS No.2(engl) p. 164-190

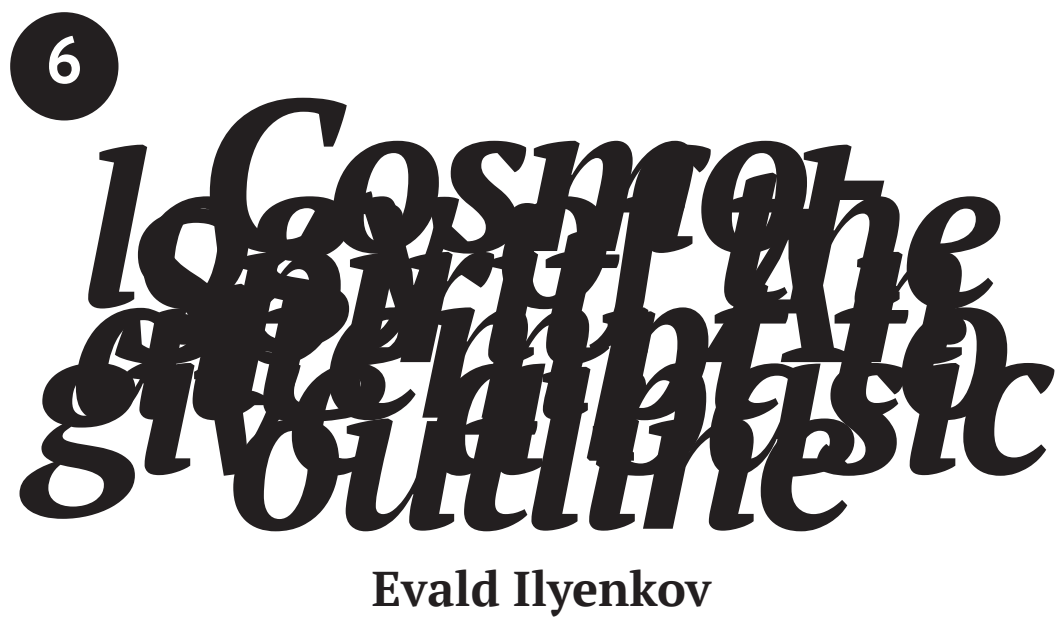

\title{
Cosmology of the Spirit
}

An attempt to give a basic outline of the objective role of thinking matter in the system of universal interaction (A Philosophical-Poetic Phantasmagoria based on the principles of dialectical materialism)

\begin{abstract}
Evald Ilyenkov's "Cosmology of the Spirit" was written in the 1950 s, but published posthumously only at the end of 1980 s as it was too heretical to be published during the author's lifetime. The text was heretical not because it was "dissident" or critical of the Soviet Union where the philosopher lived all his life, but because of its enormously speculative and hypothetical nature.

Addressing the physicist idea of the "thermal death of the universe," and creating an original combination of the Hegelian dialectics and Spinoza's notion of the attribute, Ilyenkov claims

that thought (and the seemingly contingent emergence of "thinking life") is a necessary attribute of matter, as it is able to prevent the terminal entropic collapse. While other works by

Ilyenkov were translated into several European languages, "Cosmology of the Spirit" is here available in English translation for the first time.
\end{abstract}

\section{Keywords}

dialectical materialism, cosmology, Hegel, Spinoza, Engels, matter, life, thought, entropy 


\section{Cosmology of the Spirit}

Committing no crime against the axioms of dialectical materialism, one can state that matter constantly possesses thought, constantly thinks itself.

This, of course, does not mean that matter in each of its particles at each moment possesses the capacity to think and thinks in its actuality. This is valid in relation to matter as a whole, as a substance, infinite in time and space.

Matter, with a necessity inherent in its nature, constantly engenders thinking creatures, constantly reproduces, now here now there, an organ of thinking - the thinking brain. And by virtue of the infinity of space, this organ thus exists in its actuality, in each finite moment of time somewhere in the fold of infinite space. Or, contrariwise, in each finite point of space (here by virtue of the infinity of time) thought is also realized sooner or later (if these words are applicable to infinite time) and each particle of matter by virtue of this, at some point in the fold of infinite time, forms an integral part of a thinking brain, that is, it thinks.

Therefore it is possible to say that, in each actual given moment of time, thought is an intrinsic property of matter-if at one point of infinite space matter destroys an organ of thought, the thinking brain, so with the same iron necessity at the same time in some other point it reproduces this organ.

The organ through which matter thinks of itself, consequentially, does not disappear at any single moment of infinite time, and thus matter constantly possesses thought as one of its attributes. It cannot forfeit it for a single moment. Moreover, one must assume that a brain that thinks in its actuality always exists in the fold of infinity simultaneously at all the stages of its development: at some points in the stage of its genesis; at others during the phase of its decline and at others again during the peak of its development and potency.

"...Matter in its eternal cycle moves according to laws which at a definite stage-now here, now there-necessarily give rise to the thinking mind in organic beings" (Engels 1974 [1883]: 475-76). In this sense, dialectical materialism restores the simple and profound statement of Bruno and Spinoza in a rational form, that in matter, taken as a whole, development lies in their actual consummation at every finite moment of time; in matter, all the stages and forms of the necessary development are, simultaneously, found in their actual realization. Taken as a whole, matter does not develop: not for a single moment can it lose a single attribute, nor can it acquire a single new attribute.

This, naturally, not only does not contradict but, on the contrary, presupposes the thesis that in each single finite sphere of its existence (however large it may be) there is always an operating dialectical development. But that which is valid for each single "finite" part of matter, is not valid in relation to matter as a whole, to matter understood as substance. 
As substance, matter cannot be represented as a simple sum of its "finite" parts, and all the theoretical principles valid for each of its finite parts then become invalid in relation to matter as a whole, in its eternal, closed in itself, big circle.

In relation to each separate finite sphere of its existence, it is true that thought arises on the basis of, and following, other simpler forms of existence of matter, and does not always exist; whereas other forms of matter exist always, constituting a necessary precondition and the conditions for the generation of thought.

But in relation to matter, as a whole, to matter understood as universal substance, this thesis is not valid. Here another thesis is valid: not only can thought not exist without matter (this is acknowledged by every materialist including by metaphysical materialists such as Holbach), but it also holds that matter cannot exist without thought, and this thesis can be shared only by a dialectic and materialist, the kind of materialist Spinoza is.

Just as there is not thought without matter, understood as substance, so there is not matter without thought, understood as its attribute.

To conceive of matter as a whole (as universal substance) deprived of thought as one of its attributes, means conceiving it inadequately, more meagre than it is in actual fact. This would mean in the theoretical definition of matter as substance (insofar as it is not only a purely gnoseological category), it would arbitrarily omit one of its universal and necessary attributional definitions. This would mean giving an invalid definition of matter as substance and reducing it to a purely gnoseological category.

Lenin, it will be recalled, believed it to be absolutely necessary to "deepen the knowledge of matter to the knowledge (to the concept) of substance," for only in this case will it forfeit its purely gnoseological sense.

And although the thesis "Just as there is no thought without matter, so there is no matter without thought" may sound unexpected, it is precisely in this thesis that the single principal distinction separating dialectical materialism (the materialism of Spinoza, Engels, and Lenin) from mechanistic materialism (the materialism of Galilei, Newton, Hobbes, Holbach) can be found. This thesis is beyond the reach of the latter.

Mechanistic materialism understands thought only as a product of matter, as one of the properties of matter-and for this very reason as a property that is more or less accidental: "That matter evolves out of itself the thinking human brain is for mechanism a pure accident, although necessarily determined, step by step, where it happens" (Engels 1974 [1883]: 490). According to this perspective, thought may not even take place-for this is only a more or less accidental exception, the product of a fortuitous combination of circumstances, without any detriment to matter in general.

"But the truth is that it is the nature of matter to advance to the evolution of thinking beings,"-Engels objects to this position-"hence this 


\section{Cosmology of the Spirit}

always necessarily occurs wherever those conditions for it are present" (1974 [1883]: 490). And these "necessary conditions" are essentially once again not ones of pure contingency-they themselves create with the same iron necessity that same universal motion, and, consequently, matter as a whole necessarily possesses thought permanently in its actuality and cannot forfeit it for a single moment of its existence in infinite time and infinite space.

Consequently, if philosophy as science considers only universal (infinite) forms of existence and the development of matter and if its scientific principles concern only those forms, then dialectical and materialist philosophy should not contain the thesis: "There is no thought without matter, but there is matter without thought." Rather it should contain another thesis, embracing an understanding of the infinite dialectics of their relation: "Just as there is no thought without matter, so there is no matter without thought." This thesis corresponds far more to a common philosophical perspective to the question, as well as to the dialectical (and not merely materialist) solution to this question.

The following point of the dialectical and materialist understanding of the problem has hitherto been little elucidated upon, but it is one upon which much is incumbent and regards an understanding of thought, of thinking matter, as the supreme (absolutely highest) form of motion and development.

Thought is undoubtedly the highest product of universal development, is the highest stage of organizing interactions, the limit of the complexity of this organization.

Not only does science not know such a form more highly organized than the thinking brain but neither can philosophy admit in principle of such a form as possible, for by doing so it would render philosophy itself impossible.

In this case the notion of a fundamental cognizability of the surrounding world breaks down, rendering any other philosophical system, apart from skepticism or a positivist form of agnosticism, impossible. If matter is in any way capable of engendering some higher form of motion than the thinking brain, a form that would be located in the same fundamental relation to the thinking brain, by which, for example, biological motion is related to chemical, then such an assumption would be completely equivalent to the acknowledgment of a sphere of activity that is fundamentally unknowable by thought.

In fact, this hypothetical form of development (one even more highly organized than a thinking brain) can't be related to the sphere of material phenomena: it would presuppose, as its historically necessary and its historically surpassed condition, overcome by development, not only a nature existing beyond, external to, and independent of thought, but also thought itself. This would be a certain form of development that would be possible only subsequent to thought and founded upon it. In other words, 
thought would have been preserved in it in terms of the "sublated," overcome, incidental and non-existent moment-after the manner of how chemical and mechanical movement in a living organism is transformed into an incidental form of its existence.

The laws of such a hypothetically postulated form of development could not be reduced to the laws of thought, nor deduced (that is, understood) proceeding from these laws. In other words, this form of development would be fundamentally unknowable by thought but-in terms of its higher organization-would have dominated thought as some kind of elusive sphere of actuality whose laws are fundamentally inconceivable.

In this way we would have returned to the advanced conception of Immanuel Kant: the world of phenomena (both those surrounding us, as well as those phenomena of thought itself) would be transformed into a form of external manifestation of a certain higher "essence" in terms of their laws-an essence that is fundamentally, as a thing-in-itself, inconceivable.

In other words, by this admission, we would have made fundamentally possible any type of mysticism and devilish drivel... We would have admitted that there still exists a certain "something" above nature and above thought, and this "something" by virtue of its supernatural complexity, would be fundamentally unknowable, and inconceivable to thought.

And it is of no import what title we use to name this higher form of development (this more complex form of organized motion) than the thinking brain-its essence would remain entirely the same, namely the essence of the idea of God, Providence, World Spirit, and so on.

And this perspective, inevitably resulting from assuming the possibility of a higher organization of motion of the world process than the thinking brain, would be equally as idealist as the absolute idealism of the Hegelian system, but it would have differed from the latter in that it would have necessarily assumed this higher reality to be inconceivable to thought. In other words, its perspective would be closest of all to the Kantian one.

In Hegel, even if a suprahuman Reason is admitted, then thought is nevertheless attributed with the capacity of developing to such a level where it, not ceasing to be thought, nonetheless remains equal in its power to that World Spirit. In logic, according to Hegel, the laws of thought nevertheless do coincide with the laws of the absolute and become correspondent to them. But this means that thought (although following a cunning path) is elevated all the same to the rank of the supreme reality. In sum, in The Phenomenology of Spirit human thinking becomes identical with the absolute, apprehending laws that absolute reason submits itself to and thereby converts itself to an embodiment of this supreme reality, becoming a form of motion, higher and more complex of which there is nothing and can no longer be anything.

And this understanding marked a step forward compared to Kant's conception. And it's clear that the admission of a more highly organized 


\section{Cosmology of the Spirit}

form of development of the universe than thought (however it be interpreted-materialistically or idealistically) is perfectly equivalent to the adoption of the thesis of the fundamental unknowability of the world, of the higher laws which it submits itself to in its existence.

Dialectical materialism-insofar as it is not a system of positivistically interpreted scientific data, but a philosophical system as a special science-is compelled to acknowledge (like any philosophical system with the exception of agnostic or skeptical ones) that the thinking brain is the supreme form of organization of matter, while thought, as the capacity of the brain-is equally the highest level that universal matter can reach in its gradual development.

Therefore, thought is the supreme product of the development of the universe. In it, in the birth of the thinking brain, universal matter attains such a degree after which all possibilities of further development "above" are exhausted-in terms of the complicated organization of forms of motion.

After that, the path can only lead "down," along the path of decomposition of this organization-to a purely biological-physiological level in the case of mental deterioration or still further-to simple chemistry in the case of the physiological death of the brain.

The path further "upward" is excluded. The thinking matter of the brain, the form of motion that emerges as thought, is the supreme and most intransgressible boundary of gradual development.

This is an entirely necessary conclusion of every scientific philosophy with the exclusion (as we've already shown) of agnosticism and skepticism-a conclusion whose compelling necessity has been recognized by all systems of scientific philosophy-the system of Spinoza or Fichte, Hegel or Engels.

The differences between materialism and idealism pass along another line of thought-along the line of interpretation of thought itself and its interaction with the material world. But in the recognition of thought as the supreme form of development of the universe, all philosophical systems coincide, because this recognition is a necessary condition of the existence and development of philosophy itself. If it were not so, then philosophy would not draw a single responsible and categorical conclusion, indeed it couldn't be considered a science at all.

Thus, the thinking brain with its ability to think is the absolute limit of development in terms of gradual development. But the gradual character of development is not the only form of development. Otherwise, it would have lead to bad infinity. Whereas the true infinity has, it will be recalled, the form of a circle, that of a big circle.

The supreme product of development returns through the path of dissolution into its lowest forms, again connected in this way to the eternal big circle of universal matter.

And this colossal big circle, not having a beginning nor an end, a big circle in which universal matter loses not one of the attributes that it pos- 
sesses, and acquires not a single new attribute, comprises, in a ring-like form, all the possible "finite" cycles of development.

The circular character of infinity is the only one that corresponds to the dialectical view. An alternative to this conception can be only an idea including within it the idea of the "beginning" and the "end" of universal development, "the first impulse," "a condition equal to itself" and the suchlike.

Thus, thought as an attribute (and, moreover, in its capacity as supreme product of universal development) is included in this eternal big circle of universal matter, all the time renewing its cycles. It appears as one of the links in the circle of development, as a link through which the whole big circle passes somehow in its entirety with a certain iron necessity.

In other words, the thinking brain appears as one of the necessary links, locking together the universal (vseobhschee) big circle of universal (mirovoj) matter. In the sense of "gradual" development this is the absolute peak point of the circle, subsequently followed by the return of matter to a more elementary and previously surpassed forms-biological, chemical, the fiery-liquid or incandescent and nebulous mass of celestial bodies, in the cold and undifferentiated, rarefied dust of the nebulae, in the gassy fog of intergalactic space, in the purely mechanical translocation of elemental particles, etc., etc.

We should immediately mention an important consequence that inevitably results from this acknowledgment of the supreme form of development. Having acknowledged the impossibility of higher forms than thought, than the thinking brain, as a theoretically necessary condition, we are also compelled to acknowledge a "low" boundary-a boundary lower than which the existence of matter turns out to be impossible.

Evidently we are far from discovering this (scientifically). But it is necessary to assume this theoretically. Assuming that any organized matter, higher and more complex than the thinking brain cannot exist due to the very nature of things, in the same way we also acknowledge an opposing limit-the boundary of simply organized matter, the absolutely simplest form of motion, relative to the "starting point" of the cycle. Otherwise one ends up with an incongruity: on the one hand, in terms of the complex organized matter and its forms of motion, a boundary is assumed; while on the other hand, in terms of the "simplification" of its organized form one assumes a bad infinity. Engels fully allows for such a condition in which all particular properties of matter disappear, while only those properties characterizing it as simple matter remain, with the assumption that such a condition is realized "in the gaseous sphere of the nebula." All substances in this condition, Engels assumes, "become merged in pure matter as such, acting only as matter, not according to their specific properties" (Engels 1974 [1883]: 522).

We should add here that contemporary physics in its attempts to reveal the simplest laws linking space, motion, and time, arrives at the idea 


\section{Cosmology of the Spirit}

of the "quantization" of space and time, with the idea of elementary space, time, and movement "quant(um)"-as that limit of divisibility from which (if one passes through it) the objective interconditionality of motion, time, and space would disappear. A particle that in reality (and not just in abstract terms) realizes the pure form of mechanical motion, a particle deprived of any properties apart from purely mechanical ones. "Mechanical" here is understood not in the sense of Newtonian physics but in the sense of the theory of relativity in its rational form, in the dialectical materialist form.

Such a particle should evidently be acknowledged, a particle deprived of chemical, electric, and other such properties. From a philosophical and theoretical perspective there is nothing "mechanistic" in this, but this is the conclusion automatically emerging from the acknowledgment of the supreme level of organization of matter. An acknowledgment of the absolutely highest form is impossible without acknowledging its opposite-the absolutely lowest, absolutely simplest form of matter and its motion.

Along with the atom disappear the chemical properties, along with the electron disappear the electrical properties of matter and somewhere, evidently, there exists a boundary that can't be crossed without contravening the mechanical properties (that is, the connection of simple transposition with the spatial and temporal characteristics of objective reality).

This state, perhaps, materializes and not in a "gassy sphere of the nebula," as Engels assumed; the gassy sphere itself, most likely, is in some way a more complicated stage of interaction, but in the form of a "field" as the absolutely lowest form of engagement with organized matter, as an actual existence of matter that can be reduced no further, as an absolute undifferentiation of its condition.

That's the second precondition of the hypotheses.

The third philosophical and theoretical prerequisite of the hypotheses is the incontrovertible point, according to which "all that exists is worthy of destruction," that each "finite" form of existence has its beginning and its end. This condition is applicable both to the present solar planetary system, as it is to the humankind inhabiting it.

It's clear that sometime in the dark future of times to come, humanity will cease to exist and that the eternal flow of motion of the Universe will, at long last, wash away and wipe out all traces of human culture. Earth itself will be scattered into the dust of cosmic space and will be dissolved into the eternal big circle of universal matter...

Before this happens (a distant and practically indifferent prospect for us) millions of years will have passed, hundreds of thousands of generations will have peopled the earth and gone to their graves. But time moves inexorably toward that time when the thinking spirit on Earth fades out, in order to be reborn again somewhere else in another part of the eternal Universe. 
This is incontrovertible from the prospect of any viewpoint. The idea that one should feel sorrow about this would be just as ludicrous as feeling sorrow about the fact that everything in the world is interrelated, that quantity is transformed into quality, that thought cannot exist without a brain and so on.

This fact, in this way, is not a ground for an outpouring of emotion but rather one of understanding.

But if from a practical point of view this fact conjures up absolute indifference in us, one which can in no way influence the activities of human life (after all an individual doesn't just fold up their arms even when they know that sooner or later they will need to abandon life), from a theoretical point of view this prospect is certainly not without interest.

One can't fail to note that, in some form or other, this problem has always dawned in human consciousness.

In a naïve and mystic formulation of this issue, known under the title of the final aims of human existence, those highest aims by which the thinking spirit is manifested in the universe and for which humankind endures such suffering and torment.

The answer, of course, has always had an ideological nuance. The realization of the highest moral aims, of moral law or, as in Hegel, the aim of self-understanding of the World Spirit: this wide variety of different variants are all known to us.

Dialectical materialism for the first time rationally sublated this formulation of this issue in such a way that it completely casts off the idea of any "aim" of the existence of the universe whatsoever, resolving the issue of the "aim" in the category of universal interaction.

Humankind with its thought hooked up to this universal interaction, is engendered within it and is developed and, at some point, disappears within it. The notion of the "highest aim" of human existence is sublated rationally in the understanding of the necessity of its genesis, development and death inside and through the medium of this universal interdependence of all forms of motion of universal matter.

The genesis and development and death of humankind is objectively predicated on this infinite system of interaction-in it, in its understanding one must search for the sense and role of humankind in the Universe-look for the solution to this question that, in its idealistic expression sounds like the issue of the highest, of the final aim of human existence.

"The historical genesis" of the history of humankind is fully explained in a rational and materialist manner by science. The biological development of a certain breed of monkey, then labor as a social form of interaction of the organism with the surrounding environment, as a process of the "self-emergence of the human," as a process characterized by self-development, reflected in ideological consciousness as "aims" immanent to humankind. 


\section{Cosmology of the Spirit}

A fully rational explanation of human self-development has been given by the teachings of Marx and Engels, their historical materialism, which did away, once and for all, with idealism in its last refuge.

Human history now emerged as a necessary process of self-development, a moving spring located within it, in the internal contradictions of its development and not requiring any transcendent or trascendental goals for its explanation.

From this point of view it would be worthwhile to sketch the prospects for the future more concretely than it has been done until now. That humanity, along with the earth, will perish at some point in time is indisputable and needs not be questioned.

The whole issue can be reduced to how precisely this will happen. What conditions make the end of humankind just as inevitable as its genesis, in the fold of universal interaction?

Here there immediately arises a doubt: Is it possible to formulate in some way several reasonable answers to this question? Is it possible to say anything about this apart from poetic fantasies?

We shall try at first to establish and summarize all the indisputably theoretical conditions of the task, so as to observe whether they are sufficient in finding a solution that is at least one iota more concrete than the general idea that, somehow or other, the end of humankind is inevitable.

The answer, naturally, can be found along the path of a more concrete analysis of that universal interaction within which the history of humankind is manifested and which ultimately decides all the more or less distant prospects of all that exists.

And so, first of all, the fate of humankind is closely linked with the future fate of Earth and (in wider terms) with the fate of the Solar system. This is, as it were, that closest link of universal interaction which directly determines the inevitable end of humankind.

Therefore most theoretical hypotheses of the end of human existence also resort to the idea that, at some point, in the darkness of the future, the sun will gradually cool down, and the reserves of heat on the planet will dwindle and so humankind will, therefore, already start its path to decline.

This idea until now remains the only conceivable one, since the end of humanity as a consequence of a tragic incidence (the clash of cosmic bodies, and so on) is not worth taking into account. For while such an incident can't be excluded, it cannot be taken as the basis for a theoretical understanding of the issue. It would be absurd to assume that the genesis of humankind is postulated on iron need, while its end is linked only to contingency. Both here and there there is a place for the dialectic, for both the former and the latter. Contingency itself should be understood in the case of the end of humankind, too, as a manifestation of necessary processes. In the idea of a pure accidental collision there are no dialectics: 
the collision of heavenly bodies is only one of the contingent events that could take place. Here one needs such a contingency which is not necessarily as such. We need to find that prospect which comes about (even if that precise, that completely unique, incident doesn't take place) through any other contingent event.

Engels, as is well known, accepted, as the main dialectical prospect, the hypothesis of the gradual cooling of the sun and the earth.

In his description the prospect appears like this: “...but inexorably the time will come when the declining warmth of the sun will no longer suffice to melt the ice thrusting itself forward from the poles; when the human race, crowding more and more about the eqautor, will finally no longer find even there enough heat for life; when gradually even the last trace of organic life will vanish; and the earth, an extinct frozen globe like the moon, will circle in deepest darkness and in an ever narrower orbit about the equally extinct sun, and at last fall into it" (Engels 1974 [1883]: 331-32).

The solar system, it appears, can expect precisely this prospect and humankind, considering this in an abstract manner, must share this very fate with it.

This is a necessary conclusion following from a conception of the place of the human within the immediate environment of its existence, within the immediate environment of universal interaction.

But a question arises-aren't there any circumstantial facts that overide this abstract possibility? Hasn't this prospect not been sketched out in rather too abstract a manner?

The fact that the sun and the planets will cool in time is indisputable. Yet surely humankind (and in the future this will be all the more true) will cease to be the obedient toy of external circumstances. Its power increases from year to year. Humankind will find ever newer, ever more contemporary means to liberate the reserves of heat, movement, energy accumulated in other forms apart from direct solar radiation.

The further humankind develops, the more and the deeper the energy hoards (the deeper they are hidden and the more concentrated they are accumulated, the more powerful they are) will be revealed before it, transforming them into the condition for its existence...

And doesn't the prospect of the end of humankind from the lack of direct solar radiation appear ludicrous?

Doesn't the following prospect appear ludicrous:

-Humankind uses energy to an increasingly full degree along with internal atomic motion (and the trend is to ever more elementary) structures, and the more it penetrates "into the depths" of matter, the more energy it will release from there, becoming ever more independent from the "ready" solar warmth, and on the other hand,

-it would perish precisely from the lack of direct "ready" solar warmth, it would (precisely at the height of its powers), freeze, like a helpless puppy on the frosted-up planet... 


\section{Cosmology of the Spirit}

Does not the development of productive power of humankind remove the danger of perishing from cosmic freezing, from the cold of intergalactic space?

In any case, as a tendency of its development, human power over internal structures of matter and over the motional energy enclosed within them is directly opposed to the prospect of perishing from a lack of energy, movement, warmth.

The tendency of external nature is to deprive humans of the possibility of using the "ready" warmth (not created by them) of the sun. But humans themselves create the conditions of their existence, and the "heat" received by them from the core of matter constitutes no exception. This is also a condition of human existence, created by the very existence of humankind, and without this condition, human existence would have no place in nature.

Therefore the prospect drawn by Engels during the last century, in the light of most recent developments of humankind seems rather abstract, and, therefore, invalid.

It would be a complete absurdity if humankind (by now possessing endonuclear supplies of energy) in a million years were to become helpless before the cold, through a simple lack of heat.

It is true that it will receive less and less ready external heat. But the more it will produce itself, extracting "from within" matter its concentrated reserves which (and this is theoretically indisputable) are absolutely infinite in their most minute ice-covered particles, swirling through intergalactic space.

After all, energy emitted from the sun is not lost without a trace-it accrues, accumulates in other forms and one simply needs to find the capability of extracting it.

And there is no doubt that humankind (especially under the threat of perishing from the cold) would be able to do this. Even now, when the threat of the sun's cooling is a very distant one in practical terms, it has already made considerable advances in this regard. One can imagine what it will be capable of in the millions of years remaining before that point in time! And it is worth taking this factor into account in order to abandon the above mentioned hypothesis.

Humanity, clearly, will not perish from those prospects initially depicted (neither from the cold, nor from a simple lack of warmth). It is evident that one needs to reject such a presupposition.

But so far we have demolished the only reasoned hypothesis: a conjecture based upon an understanding of the place of humans in the fold of universal reciprocity, and have offered no new hypothesis in its place. In the same way one needs to jettison the idea that humankind will find its end as a result of physiological degeneration, physiological regression. Physiology is that very same nature, and humans are moving toward a greater and greater power over the nature of the given matter of its activity. 
Extracting energy accumulated from within the elementary particles, freely turning one form of motion into others, one chemical element into others, both more as well as less complex than the original ones and governing at the same time one's own physiological development, directing it through a viable course (from the point of view of new conditions), humankind has, evidently, all the potential to escape the fate of freezing to death, or that of a "cold" and hungry death...

It has, it seems, the strength to create (at least in a small part of space) an artificial environment and support it, preserving and reproducing it without the aid of the generous and giving energy of the sun.

By now this has become a fully outlined trend in human development.

But that which humankind (thinking matter in general) is not in a condition to endure, in spite of its power over nature (whatever the level that this power reaches) is the opposite state of universal matter to the cold of intergalactic space, a state toward which the evolution of the world leads it just as inevitably as to that of cooling: the fiery-incandescent "youth" of cosmic matter, the state of incandescent gas of the young, engendered nebula, a starting point for a new cosmic cycle.

This fiery-vaporous condition in which all elements are transformed into wildly rotating vortices and where it is fundamentally impossible to preserve any artificially created borders at all, from behind which humans could hide, no arbitrarily durable and heat-resistant "husk," capable of separating an artificial environment from that which remains, from the "non-humanized" world, evidently turns out to be that absolute border beyond which the existence of thinking matter is already impossible. Maybe humankind is able to avoid death in the ice-covered planet. This is a prospect that is fundamentally possible.

But no efforts will save it from death in the hurricane of global "fire" that, at some point, will return the vulcanic youth to our global island.

Thus we should probably see such an absolute boundary in the state of incandescent vapors to which any cosmic system will inevitably pass through in the course of a big circle, if the frozen nature of the cooling universal space does not represent this absolute boundary for the existence of thinking matter. This, of course, does not exclude in any way the prospect that in certain cases it too may be the direct cause of the death of humankind, just as the accidental tragic collision of celestial bodies may also be.

The demise, perishing, end, disappearance of thinking matter also remains, in this case, irreversible-the principles of dialectics and materialism are fully preserved here too. But the concrete picture of this end turns out to be rather different. Above all, the boundaries of the existence of thinking matter extends somewhat in time. The inevitable end will come from this perspective somewhat later (although this "somewhat later" in reality means in excess of millions of years), and for this supple- 


\section{Cosmology of the Spirit}

mentary period, humankind, undoubtedly, can reinforce, even further, its power over nature, and reach such peaks of power that it is presently impossible to imagine even with the aid of the most unrestrained poetical fancy.

But (and this is central) a theoretically very significant circumstance is effectively included among the conditions for solving the problem, about which one could fail to note when presupposing that humankind would perish from the cold in the freezing earth hovering around an icecovered sun, but which now returns to the foreground. This is a question about the circumstances at which the cooling universal matter will necessarily transform itself into a state of incandescent nebula, turning into a massive hurricane, heated up to millions of degrees Celsius, and gathering at its center all the dispersed radiated reserves of motion and thereby giving new life to the universal matter of cosmic space, expiring in the icy desert of the so-called thermal death.

The origin of this new cycle of development of cosmic matter is the point in which the dispersed matter by means of irradiated stars and their intrinsic motion, have again, in some way, concentrated in the form of an incandescent rotating nebula, concentrating at its center all the particles and motional energy previously dispersed in space: this turns out to be the absolute boundary in which all conditions under which the thinking spirit can exist, inevitably disappear.

The end of thinking matter coincides, in time and according to circumstances, with the beginning of a new developmental cycle of matter in cosmic expanses-from the point in which the fiery rebirth of dying worlds takes place.

This point in which matter and motion, irretrievably lost thanks to their emission, in some way once again becomes concentrated, and accumulates in the form of incandescent clots, hurricane-like whirling gas and vapors, proves to be that point in which thinking matter must disappear in an absolutely obligatory manner.

But that same question about the concrete picture of the end of humankind, the disappearance of thinking matter, is raised in connection with those natural conditions, in which the process whereby worlds dying from "thermal death" are reborn to a new life becomes possible and even inevitable.

In other words, the conditions of a fiery rebirth of cosmic systems turn out to be, simultaneously, the conditions under which the death of thinking matter, the death of thinking spirit, finally becomes absolutely inevitable.

Both problems effectively merge into one.

And the most interesting aspect is that each of these issues considered separately, one abstracted from the other, is still unresolved by science, and perhaps (and in this lies our hypothesis) are fundamentally unresolvable using such an approach. 
We have established that the question of the death of the thinking brain cannot be resolved outside the conditions created by the development of the cosmic systems, within which flow the history of thinking spirit's development, and we have come to the conclusion that the absolute inevitability of this death coincides with the beginning of the fiery rebirth of worlds perishing from "thermal death."

We shall look at the question now from another perspective-from that of the particular destinies of cosmic systems.

Does it not seem that this problem is fundamentally unresolvable unless we investigate those factors introduced by the course of the universal process of thinking spirit, those conditions which are created with their indispensable participation?

In other words, does it not seem that both this and the other process are impossible to understand without considering their interaction with the other? Does it not seem that the process of the fiery rebirth of worlds, expiring in the conditions of "thermal death" at the same time cannot be understood without considering the active role of the thinking spirit in the big universal circle, in precisely the same way as the death of the spirit cannot be understood outside its links to this cosmic process?

We shall analyze in a more detailed manner the conditions of the theoretical task proceeding this time not from problems of thought, but from cosmic conditions themselves, from the pure immanent laws of selfdevelopment and the death of the cosmic systems within which is born, blossoms, and then fades the highest creation of the universe-its thinking spirit.

That the fate of the thinking spirit is conditioned by the fates of wider (cosmic) processes, this too we thereby set down at the very basis of our hypothesis.

But it is here, precisely, that we find ourselves before an issue which hitherto has been unresolved (and perhaps unresolvable from that perspective from which it has viewed up until now).

This is the problem of the so-called thermal death of the Universe. This problem may be briefly expressed in the following way.

All the celestial bodies and systems known to science, gradually (through emission) lose the reserves of their internal energy, and lose them irrevocably, gradually cooling in the vain attempt to heat at least to a billionth part of a degree of its surrounding environment.

In so doing, the moving matter of the warmed celestial bodies disperse equally into intergalactic space, turning into cold vapors formed of ice, the temperature of which is comparable to absolute zero and only differs from it in its vanishingly small size.

The process connected with the emission of heat in the universal space, can, at this point, be deemed irrevocable, moreover absolutely irrevocable, so that it seems that there is a tendency toward the fact that the entire universal matter with its intrinsic motion is shared in abso- 


\section{Cosmology of the Spirit}

lutely equal parts in the intergalactic sphere and the entire Universe, which as a whole, gradually moves toward a condition of "thermal death," that is, to that stable equilibrium that excludes every possibility of a reverse transition toward a differentiated state.

At the end of last century Rudolf Clausius even calculated that 453 out of 454 parts of the active motional energy of universal matter has already been lost on its path so far. ${ }^{1}$ All the remaining share of active energy has already, according to his calculations, been transformed into a highly concentrated state, into the singular state "equal to itself" of "thermal death."

From the philosophical and theoretical perspective this is, as Engels has already demonstrated, an absurdity that presupposes "the origin of the world." But hitherto the reverse process has not been revealed nor explained. Only one thing is clear-if it had not taken place somewhere and in some way, then the Universe as a whole could not have existed and, on account of the infinity of time, it would already have long ago turned into an undifferentiated nebula, whose temperature in all its parts would be absolutely equal and whose motion absolutely equally shared among all particles of matter, each of which would thus be practically immobile, and would not interact with neighbours in any other way, except in a purely mechanical one...

Only the process that has a tendency to precisely such a lifeless state of universal matter is known to us, whereas the reverse trend, contradicting the above process, is unknown to us-a process by means of which the reverse reallocation of motion takes place in the Universe- although theoretically it is absolutely clear that such a process does exist, indeed, its existence is not in doubt.

Substantially, things can be represented like this:

...with the exception of an infinitesimal portion, the heat of the innumerable suns of our island universe vanishes into space and fails to raise the temperature of universal space even by a millionth of a degree Centigrade. What becomes of all this enormous quantity of heat? Is it for ever dissipated in the attempt to heat universal space, has it ceased to exist practically, and does it only continue to exist theoretically, in the fact that universal space has become warmer by a decimal fraction of a degree beginning with ten or more noughts? (Engels 1974 [1883]: 334).

It is clear in theory that this is not the case, that the equally cooled matter of intergalactic space into which any celestial body will gradually be transformed into, due to emission, in some way (and this way can only

1 Actually Frederick Engels states that it was Helmann von Helmholtz who calculated this amount. 
be a natural way), concentrates, in a reverse way, in clusters of highly incandescent gas. In so doing it engenders new stars, new worlds, new planetary systems.

But how this happens in a real and concrete way, in what manner, still remains an open mystery.

A theoretical issue which, as Engels demonstrated, can only be resolved provided that it is clearly set out and that the way it is set out assumes a dialectical materialist view on things. From the perspective of materialist dialectics the question must and can only be set out in this way:

...when it has been shown how the heat radiated into universal space becomes utilizable again. The theory of the transformation of motion puts this question categorically, and it cannot be got over by postponing the answer or by evasion. That, however, with the posing of the question, the conditions for its solution are simultaneously given"-this is selfevident (Engels 1974 [1883]: 562).

We have formulated this condition above: the solution must be based on theoretically indisputable conditions, that the "reverse" process-the process of a concentration of dispersed motion in clusters of incandescent gas-taking place in some way, somewhere in some permanent manner in the fold of the Universe and comprising its constant inner condition of existence. And the whole issue consists in establishing, in discovering this process.

No wonder that it has not yet been solved-Engels continues-it may still be a long time before we arrive at a solution with our small means. But it will be solved. Just as surely as it is certain that there are no miracles in nature and that the original heat of the nebular ball is not communicated to it miraculously from outside the universe (Engels 1974 [1883]: 562).

We should note that even nowadays, in the middle of the twentieth century this question has still not been resolved just as it had not been at the end of the nineteenth century.

The general statement that the general quantity (die Masse) of motion is infinite, that is, inexhaustible, is also ineffective in overcoming our difficulties in each separate case. Here too, following such a path, we will also not arrive at the rebirth of dying worlds, with the exclusion of those cases provided for in the aforementioned hypotheses and which are always connected to the loss of energy, that is, only temporary cases. The big circle here is not arrived at and will not be arrived at, until such a time that it reveals the potential for a new use of emitted heat (Engels 1974 [1883]: 562). This question, thus, should not concern itself with individual 


\section{Cosmology of the Spirit}

cases but must be resolved in connection with the universal (vseobshchee) big circle of universal (mirovoi) matter. This big circle in itself, within itself, within its attributive and necessary cycles must necessarily lead to the rebirth of dying worlds in the form of incandescent nebula.

Therefore, one should seek to solve the riddle not only in a concrete and physical (concrete-astronomical) form but also in a general philosophical form. In other words, the possibility and necessity of such a rebirth should be demonstrated and sought within, in the attributive and necessary form of the existence of universal matter-not outside it and not in contingent events which concern only single cases.

For in these separate cases the problem may well even be resolved, but it still remains unresolved in a general sense.

Thus the problem, in general terms, can be encapsulated like this: physics and astronomy thus far hold data concerning the process of dispersion of matter and the motion of stellar bodies- a process tending to lead to the state of so-called thermal death. The concept of so-called thermal death is nothing other than the theoretical expression of the tendency of the process connected with the emission of heat and light in intergalactic space.

But natural science research has still not yet demonstrated the reverse process - the process of the rebirth of dying worlds, the process of the transformation of ice-covered steam of intergalactic space into incandescent nebula.

An indisputable theoretical conclusion is that such a process through some spontaneous way intrinsic to the very nature of moving matter is continually taking place. Without such a process, the existing Universe could not be preserved and reproduced in eternity in a natural way, it represents an absolute necessity, inherently posited by the motion of universal matter, a condition for the existence of the Universe.

Without this process then there exists "god," "the origin of the universe," "the first impulse" leading matter from a practically immobile state of "thermal death" and all such devilish drivel and mysticism.

Apart from this, the idea that the "entropy of the world" "cannot be eliminated in a natural way, but, on the contrary, can be created" (this is, the idea of "thermal death" expressed in terms of thermodynamics), is equivalent to the denial of the universal law of the preservation and transformation of energy. This idea assumes, as Engels has shown, that energy, active motion, is dispersed if it is not quantitative but qualitative.

The law of preservation and transformation of energy assumes that energy can only be preserved in the course of its qualitative transformations and this course cannot be a one-sided process, irrevocable in any one of its links. All forms of motion of matter, in some way or other, reciprocally transforms itself into others, they are reciprocally reversible. If this had not been the case, then the Universe existing today would not have been able to exist without the constant interference of supernatural 
forces, while the law of preservation of matter and motion would have turned into a fiction.

Therefore the whole problem consists of explaining and showing through what path, by what natural way, can the heat emitted into universal space be used; where and how this dispersed emission of matter and motion once again accumulates in such a form which is capable, conversely, of transforming itself into extraordinarily heated and compact agglomerations, in world islands of incandescent gas drawing to its center all practically "immobile" matter dispersed into the surrounding spaces and building from it its body-a body of future stars, suns, planetary systems, and so on.

Here we will allow ourselves to state our hypothetical thesis regarding the where and the how of this process, which regularly returns universal matter from the state of "thermal death" into a state of incandescent clouds of gas, and is carried out with a necessity inherent in the very nature of moving matter.

The hypothesis consists of the following.

Why not presuppose that this reverse process takes place with the participation of thinking matter, thinking spirit-as one of the attributes of universal matter-and that without its contribution, without its help, this process would be impossible and inconceivable?

This conjecture does not affect or undermine (even in the most minimal way) a single, even the most insignificant, principle of materialism and materialist dialectics.

In actual fact, the thinking spirit remains the highest product of matter's development, its necessary generation, its attribute.

The development of the brain's thinking matter remains intertwined in the chain of universal material interaction and it is contingent upon and defined by this interaction in general and as a whole.

Matter (as substance), in line with this presupposition, remains, according to the nature of things, primary. The necessary processes of its development, to some degree, engender the thinking brain as an attribute.

The thinking matter of the brain-as the supreme form of motion of universal matter-doesn't engender anything supernatural. On the contrary, its death appears as a simple trasformation into other, more elementary forms, of motion, its death emerges as the engendering of new forms of motion of matter.

The only new aspects, to which our hypothesis leads us, consist solely in the fact that the death of thinking matter is necessarily connected to the transformation of cooling matter of intergalactic space into an incandescent nebula, and represents a necessary factor in this latter process.

Nothing anti-materialistic, nor even non-materialistic is brought into the concept of this process by this hypothesis. Thought itself is a natural process and there is nothing surprising in that it, as such, takes place within other natural processes and in turn it actively affects their course. 


\section{Cosmology of the Spirit}

Indeed dialectical and historical materialism in no way deny the reverse impact of thought on material processes. In this given case we are dealing with one of the concrete forms of such a reverse active impact. Nothing more.

In this way all the principles of dialectics and materialism not only are not brought into question but, on the contrary, are posited at the foundation of the hypothesis.

Moreover, a whole array of philosophical and theoretical tenets of dialectical materialism acquire under these conditions a rather more concrete form of expression, not to mention the fact that the question of the "entropy of the world" is essentially resolved.

In actual fact, if the thinking matter of the brain is the same matter, then thought (observed not in its narrow gnoseological aspect but in terms of its place and role among other forms of motion and development of matter) is also a form of the motion of matter, moreover, it is its supreme form (motion is "not merely change of place; in fields higher than mechanics it is also change of quality” [Engels 1974 (1883): 531]), then there is nothing out of bounds in the fact that thought is regarded (from the perspective of the universal process of the quantitative and qualititative transformation of some forms of motion into others) as one of the links of a universal (vseobshchee) big circle of universal (mirovoj) matter, as one of the forms into which all other forms transform themselves and which, in reverse, turns into other forms or contributes to their reciprocal transformation.

In these circumstances, just as the thinking matter of the brain is the supreme product of universal development so it is reasonable to assume that in the course of the universal big circle, in the mutual transformations of one form of motion of universal matter into others, it has a special place and plays a special role-such a role which cannot be played by other, less complexly organized forms of motion. And this special role, befitting its place in the system of forms of motion of universal matter (as the supreme form of motion) is outlined in our hypothesis.

In real terms this role can be represented thus: Humankind (or some other combination of thinking beings) at some, very high, point of its development-at the point acquired when matter, of more or less vast cosmic spaces within which humankind exists, starts to cool and is close to the condition of so-called thermal death; in this fateful point for matter and in which in some way or other (unknown to us, of course, living at the dawn of the history of human power), consciously facilitates the start of the reverse (in comparison with dispersed motion) process, a process transforming the dying, freezing worlds into the fiery- incandescent hurricane of emerging nebula.

In these conditions, the thinking spirit sacrifices itself and, in this process, is not able to preserve itself. But its sacrifice takes place in the name of its duty to Mother Nature. The human, a thinking spirit, returns 
its old debt to nature. At some point, in its youth, nature engendered thinking spirit. Now, on the contrary, the thinking spirit, at the cost of its own existence, returns to mother nature, dying of "thermal death," a new incandescent youth -a state in which it is able once again to start colossal development cycles, which at some point again, at a different point in time and space will once more lead to the emergence of a new thinking brain, a new thinking spirit from its cooling core...

From this perspective the definition of thought as an actual attribute (and not only "mode") of matter is understood.

Contrariwise, thought cannot be qualified as attribute.

Indeed the concept of an attribute includes the idea that the given form of motion of matter represents an absolutely necessary product of its existence-therefore an absolutely necessary condition (not able to disappear) of its infinite existence.

In other words, a characteristic of thought as attribute presupposes that it (as the highest form of motion) is an absolutely necessary link through which matter passes all the time, again and again, in each of its finite cycles of its colossal big circle-such a form, which this big circle reproduces again and again with an iron necessity, intrinsic to its nature.

Consequently, the appearance of the thinking spirit within the framework of the big universal circle is not at all something accidental that, pari passu, could simply not exist, but an internal and assumed condition of its own realization. Otherwise it is not an attribute but only a "mode."

Indeed, if one assumes that the thinking spirit is born somewhere on the periphery of universal matter only in order for it soon to vanish fruitlessly and without a trace, flaring up for a brief moment on a cooling planet only in order to be extinguished again, leaving in its wake only wrecks of material culture that just as quickly disperse in the Universe the stream of its perpetual motion-if one assumes such a fate for the thinking spirit, then one ends up with a very strange notion of "attribute."

Indeed in this case thought turns out to be something like mould on a cooling planet, something like the senile disease of matter, and certainly not the highest flower of creation, not the highest product of universalworld development.

In this case thought, even if it is still called the "the highest flowering" of matter, the flower turns out to be barren-a beautiful but absolutely infertile flower, blossoming somewhere on the periphery of universal development only in order to instantly fade under the icy or the fieryincandescent gust of a hurricane from the infinite Universe [...] All the effective development of universal matter in this case takes place alongside its development, completely autonomous from it, and its appearance has absolutely no influence on the destiny of universal development.

Thought turns into an absolutely infertile episode which, pari passu, would also not have happened at all without any detriment to everything else. 


\section{Cosmology of the Spirit}

Hardly does such a role correspond with the place of thought in the system of universal matter's form of motion. The highest form of its motion cannot be its most infertile and its most useless.

There is far more basis to assume that thinking matter, as the highest quality form of motion of universal matter, plays a by no means unimportant role in the process of the universal big circle, a role corresponding to the complexity and the eminence of its organization.

Why not assume in this case, that thought is precisely that most qualitatively peak form in which is realized the accumulation and productive use of energy, emitted by the suns?

That is, this same link which as yet is lacking, in order for an actual big circle to be possible, and not a one-sided and irrevocable process of dispersal of matter and motion in intergalactic space? Why not presuppose that matter in its development specifically creates with the aid, and in the form, of the thinking brain those same conditions, in the presence of which the emitted energy from the sun isn't fruitlessly wasted on the simple heating of universal space, but accumulates into the qualitatively highest form of its existence, and then is used like a "rigger hook," as a detonator, originating the process of reverse rebirth of dying worlds in the form of incandescent nebula?

Indeed, and in this qualitatively higher form of motion, accumulated in the form of material culture, in the form of the power of thinking beings over dead matter, in the form of thought and its products-in this qualitatively higher form of motion, it transforms a tiny fraction of heat, radiated from the suns in the cosmic space. The quantitative minuteness of this share fully compensates that which it accumulates in a qualitatively higher form-in such a way, by which nature itself (without the mediation of thought) cannot transform an infertilely dissipated emitted wamth.

Humankind is already able to release those reserves of motion which, without its knowledge, would have remained connected and dead in nuclear structures, that in a hypothesis according to which the future of humankind turns out to be capable of releasing from its bound state such a quantity of energy that would be sufficient to transform the cooling matter of our cosmic island into an ocean of incandescent vapors-in this hypothesis, there is already nothing astonishing and mystical.

The material and spiritual culture of thinking beings that is very rarely manifested in nature and that requires extraordinarily specific conditions for its appearance, and proves to be that form of motion through which there takes place a concentrated accumulation of heat emitted by suns - a heat that is abortively dissipated via all other channels, and that only in this form is again used as a means, a method, of the fiery rebirth of the freezing areas of the large Universe.

In concrete terms, one can imagine it like this: At some peak point of their development, thinking beings, executing their cosmological duty 
and sacrificing themselves, produce a conscious cosmic catastropheprovoking a process, a reverse "thermal dying" of cosmic matter; that is, provoking a process leading to the rebirth of dying worlds by means of a cosmic cloud of incandescent gas and vapors.

In simple terms, thought turns out to be a necessary mediating link, thanks only to which the fiery "rejuvenation" of universal matter becomes possible; it proves to be this direct "efficient cause" that leads to the instant activation of endless reserves of interconnected motion, in a similar manner to how it currently initiates a chain reaction, artificially destroying a small quantity of the core of radioactive material.

In this given case the process, apparently, will also have a "chainlike" form, that is, a reaction, one that self-reproduces itself in a spirallike way; a reaction that creates, along its own particular course, the condition for its own flux in its expanding (at every moment) scale. Only in this given case, does the chain reaction spread not through the artificially accumulated reserves of radioactive material, but through the naturally accumulated reserves of motion of the Universe, on the reserves connected with the condition of the "thermal death" in the universal space.

In simple terms, this act materializes in the guise of a colossal cosmic explosion having a chain-like character, and the matter of which (the explosive mass) emerges as the totality of elementary structures, is dispersed by emissions through the whole universal space.

From the perspective of contemporary physics this does not appear at all inconceivable.

Indeed it is clear that the smaller the artificially destructible structure, the greater the reserves of internal energy that are released during its destruction. The destruction of chemical structures (which happens by simply burning them) gives a comparatively small dose of released energy. An incomparably greater quantity of energy is released with the destruction of the atomic core. The "simpler" the structure to be destroyed, the greater the quantity of energy that is extracted, indicating that the smaller and simpler the structure of matter, the more durable its inner connections, then the harder it is to destroy but the greater the energy it obtains in the case that one manages to provoke a chain reaction.

If one can draw the prospect of the development of science and techonology in the future, then the trend is clear: Humankind is moving in the direction of a chain destruction of ever simpler and, at the same time, ever more durable structures of matter, releasing in the process a greater and greater quantity of energy connected with these structures. And however large the loss of energy needed to destroy the first particle, that is, to initiate this chain reaction, this loss does not compare at all to the general quantity of motion extracted during the chain reaction.

And the theoretical prospect is the following: an infinitely small structural unit of matter would, in exchange, obtain a proportionately infinite quantity of energy during its release-a quantity that is sufficient to 


\section{Cosmology of the Spirit}

destroy and transform into incandescent vapors an infinitely large mass of cooling matter.

So the old formulation of the existence of the law of the preservation of matter and motion stated by Leibnitz, finds its confirmation in a new light: If a minute speck of dust were destroyed then the whole Universe would collapse. The whole infinite Universe cannot, of course, be destroyed by such an act, but, in so far as the destroyed structure in terms of its measure and complexity of organization tends to a vanishingly small limit, then the quantity of energy released in this case tends to infinity. The field of universal matter, grasped by the process and included in the chain reaction, remains therefore limited by some border. What these limits are it is, of course, impossible to say now, just as it is impossible to indicate the measure and qualitative characteristics of that particle, whose destruction is necessary for it to trigger this process. But this process fully explains the possibility of transforming an arbitrarily large final mass of cooling matter into an incandescent nebula capable of engendering a new world.

From this perspective, apparently, the hypothesis bears up to fundamental criticism.

Thought, as a result, also emerges as the very link in the universal big circle, through which the development of universal matter is contained in this form of the big circle-in an image of a snake biting its tail, as Hegel loved to express the image of true (as opposed to "bad") infinity.

The task, in this way, has been resolved while observing all the conditions. Not one principle of materialism has been affected. Several theses of dialectics have acquired an even more concrete form of expression. Thought conceived as an actual attribute of matter, as the highest product of universal development, as the highest creation of matter that blossoms with necessity in its fold and in addition gives the necessary fruits from the perspective of universal development. The law of preservation and transformation of matter and motion has also been observed and concretely realized. And along with this, a possible path has been shown, in which the use of emitted heat by the stars takes place to reverse the process - a process of the concentration of matter and motion in a toughened and heated nebula, in an incandescent rotating mass of gas. But that which is no less important and interesting from the perspective of the reciprocity of matter and thought-thought, the thinking spirit, is assigned by this hypothesis such a role in the course of the universal big circle, which to a far greater degree corresponds to its position on the ladder of development, than the notion corresponding to which all the development of spiritual and material culture, all the history of the thinking spirit leads to a zero outcome, to simple death, leaving no remaining traces.

The hypothesis premised on the place and role that the thinking spirit necessarily plays in the system of universal interaction of universal 
matter, taking account of objective and other circumstances emerging in the universe that reach beyond will and consciousness, providing an insight into that same "highest" and "final" goal of the existence of the thinking spirit in the system of the universe, upon which all, and every kind of religion, has always speculated. This "final goal" itself is understood as inherently an achievable consciousness, reflecting the place of the thinking spirit in the system of objective conditions assumed by the development of universal matter.

And this, objectively derived, "goal" is endlessly greater and more majestic than all those pathetic fantasies that have been invented by religions and those philosophic systems linked with them.

The highest and final goal of the existence of the thinking spirit turns out to be cosmic and grand as well as sublime and wonderful. It differs from other hypotheses concerning the end of humankind not in that, through this end, it puts to an end universal death (perishing, death, destruction represent an absolutely necessary result in any hypothesis); but only that death is outlined by it not as a senseless and fruitless end but as an act which in its essence is a creative one, as a prelude to a new cycle of life for the Universe.

Such a meaning for humankind and such significance of its death cannot, evidently, be recognized in any other hypothesis.

Death, indeed, is all the same inevitable and its inevitability cannot not be recognized by any hypothesis in this. And the single difference between the possible hypotheses consists only of the different interpretations of objective meaning and the role of the act of perishing in the fold of the universal big circle of universal matter, the place and role of this act in the system of universal interaction.

The suggested hypothesis sets itself apart with the advantage that the death of humankind (and the thinking spirit in general) stands in this light not as senseless, as in any other possible hypothesis, but as justifed as an absolutely necessary act from the perspective of the universal (vseobshchee) big circle of universal (mirovoj) matter, developing according to its objective laws.

This being said, thought remains a historically transitional episode in the development of the universe, a derivative ("secondary") product of the development of matter, but a product that is absolutely necessary: a consequence that simultaneously becomes the condition for the existence of infinite matter.

In regard to matter and thought there appears an effective dialectics - a reciprocal dependence, inside of which matter, although it remains primary and determinant (the first in nature), nonetheless turns out as conditional upon the reverse active impact of thought.

Thought turns out to be an actual attribute and the thesis: "Just as there is no thought without matter, so there is no matter without thought" acquires a real and concrete sense. 


\section{Cosmology of the Spirit}

In this light thought appears as not only the highest and most wonderful flower of the universe, but also a flower which is not infertile, as a flower that through its death, engenders an absolutely necessary fruit, resulting from the perspective of the universal big circle.

The death of the thinking spirit becomes a genuinely creative act, an action that transforms the freezing deserts of intergalactic space, immersed in gloom, into rotating masses of incandescent, bright, warm sunny worlds; a system that becomes the cradle of new life, a new dawn of the thinking spirit, immortal like matter itself...

The death of the thinking spirit becomes, in this way, its immortality. And somewhere again-in the endlessly distant future-new beings in which nature evolves a thinking spirit, will (like us today) contemplate the worlds of stars, shining in the sky of their earth, with the proud consciousness that these worlds owe their existence to a once vanishing thinking spirit, its great and fine victim.

In the radiance of the starry sky, the thinking being will see a testimony to the power and beauty of the immortal even in the death of its thinking spirit-objectified, sensually perceptible, but not calling into any doubt its own power over the sensible world.

The starry sky, just like the entire surrounding nature, will be for the thinking being a mirror in which it will reflect its own infinite nature. Through the glowing of the stars, the thinking spirit will be spoken to (in a language understood only by him), the immortal thinking spirit eternally revived in its products.

And in the contemplation of eternal nature, man (like every thinking being) will feel a pride in itself, of the cosmic scale of its own universal and historical mission; the place and role of the thinking being in the system of universal interaction.

Conscious of the colossal scale of its role in the universe, the human being discovers also the high sensation of their highest purpose; the highest goals of its existence in the world. Its activity is filled with new pathos, before which the pathetic pathos of religion will fade.

This will be a pathos of truth, a pathos of the true consciousness of its objective role in the universal system.

It is clear that to fulfill its universal and historical mission, the thinking spirit will find this condition only during the peak of its development, of its power; to that point which we, people of the twentieth century will obviously not live to see. Millions of years will pass, thousands of generations will be born and go to their graves, a genuine human system will be established on Earth, with the conditions for activity-a classless society, spiritual and material culture will abundantly blossom, with the aid of, and on the basis of, which humankind can only fulfill its great sacrificial duty before nature.

For us, for people living at the dawn of human prosperity, the struggle for this future will remain the only real form of service to the highest 
aims of the thinking spirit. And to the struggle undertaken today, to the activity nowadays which fulfills our hypothesis, nothing is added to and nothing is removed from it; adding only the proud (and having, for now, a purely aesthetic character) consciousness that the activity of humankind is made spiritual not only by the pathos of "final" human goals, but the fact that it has, as well as these, also a universal and historical meaning, and materializes immortal goals determined by the entire system of universal interaction.

And in the light of this hypothesis set forth in a completely new way, the genial words of "Dialectics of Nature" sound with even greater prophetic strength:

...but here either we must have recourse to a creator or we are forced to the conclusion that the incandescent raw material for the solar systems of our universe was produced in a natural way by transformations of motion which are by nature inherent in moving matter, and the conditions for which, therefore, must also be reproduced by matter, even if only after millions and millions of years and more or less by chance, but with the necessity that is also inherent in chance (Engels 1974 [1883]: 333).

And with the aid of our hypothesis we acquire a new basis for our certainty in that:

Matter remains eternally the same in all its transformations, that none of its attributes can ever be lost, and, therefore, also, that with the same iron necessity that it will exterminate its highest creation, the thinking mind, it must somewhere else and at another time again produce it (Engels 1974 [1883]: 335).

And therefore, we can add, that the thinking spirit is not a barren flower that blossoms for a short moment only to fade again almost immediately, but is just as much a condition for the existence of matter as it is a necessary consequence of it, that is an intrinsically posited, infinite and universal (vseobshchee) condition of the objective reality of universal (mirovoj) matter, the actual attribute of matter as an infinite substance of the universe.

Translated from the Russian by Giuliano Vivaldi

\section{Bibliography}

Hegel, G. W. F. (1977). Phenomenology of Spirit. Trans A. V. Miller. Oxford: Clarendon Press.

Engels, Friedrich (1974). “Dialectics of Nature” [1883]. In Marx \& Engels Collected Works, Vol. 25, 313-590. New York: International Publishers. 